\title{
Arbeitgeberverbände des öffentlichen Sektors
}

\author{
Berndt Keller
}

\section{Einleitung und Problemstellung}

Die Arbeitsbeziehungen des öffentlichen Dienstes (im Folgenden ÖD) sind wie die der Privatwirtschaft ,dualer" Natur; sie sind allerdings durch relevante Besonderheiten gekennzeichnet (Keller/Henneberger 1999). Die beiden Ebenen sind formalrechtlich getrennt, weisen aber faktisch enge Beziehungen bzw. wechselseitige Abhängigkeiten auf. Auf der betrieblichen bzw. Dienststellenebene stellen Personalräte, die über eine eigenständige gesetzliche Grundlage in Form der Personalvertretungsgesetze von Bund und Ländern verfügen, das funktionale Äquivalent zu den Betriebsräten der Privatwirtschaft dar (Keller/ Schnell 2003, 2005). Auf der überbetrieblich-sektoralen Ebene bestehen auf Seiten der Arbeitnehmer Gewerkschaften und Interessenverbände, deren Dachverbände der Deutsche Gewerkschaftsbund (DGB) und der Deutsche Beamtenbund (DBB) sind. Die Informationen und Kenntnisse über Arbeitgeber(verbände) als korporative Akteure der Arbeitsbeziehungen sind nach wie vor lückenhaft, was u. a. auf deren defensive Informationspolitik und mangelnde Öffentlichkeitsarbeit zurückzuführen ist. Auch die seit den frühen 1990er Jahren intensiv geführte Diskussion um die Einführung neuer Steuerungsmodelle bzw. eines New Public Management (Naschold/Bogumil 2000) hat diese Wissenslücke nicht beseitigt.

Dieses Kapitel stellt einen Beitrag zum Abbau dieses Defizits dar. Neben einer Reihe von Gemeinsamkeiten der Arbeitgeberverbände in Privatwirtschaft und ÖD bestehen wesentliche Eigenheiten, die sich durch die spezifischen rechtlich-institutionellen Rahmenbedingungen erklären lassen. Im Mittelpunkt stehen die kommunalen Arbeitgeber, weil auf dieser Ebene wegen der vergleichsweise großen Zahl korporativer Akteure am ehesten verbandliche Strukturen vorhanden sind (Abschnitt 2). Auf der Ebene der Länder besteht eine Arbeitgebervereinigung, die wir ebenfalls kurz behandeln (Abschnitt 3). Auf der Ebene des Bundes ist traditionell der Bundesminister des Innern mit der Interessenwahrnehmung beauftragt (Abschnitt 4). Nach der Behandlung der „logic of membership“ geht es um theoretische Erklärungen (Abschnitt 5) sowie um die „logic of influence“ (Abschnitt 6).

Von entscheidender Bedeutung für die Organisation der Arbeitgeber und damit für die Arbeitsbeziehungen des ÖD insgesamt (Keller 1983, 1993) ist der Rechtsstatus der Beschäftigtengruppen mit dem „Dualismus“ des privatrechtlichen Arbeitnehmerstatus von Angestellten und Arbeitern und öffentlich-rechtlichen Dienstverhältnisses der Beamten:

- Die Arbeitsbedingungen von Angestellten und Arbeitern werden wie in der Privatwirtschaft durch das Arbeitsrecht, vor allem durch Tarifverträge, geregelt, die die Arbeitgeber(verbände) mit den zuständigen Gewerkschaften abschließen. ${ }^{1}$

1 Dabei ist traditionell vor allem zu unterscheiden zwischen Lohn- bzw. Vergütungstarifverträgen und Manteltarifverträgen (u. a. Bundesangestelltentarifvertrag - BAT, Manteltarifvertrag für die Arbeiter des Bundes - MTB II, der Länder - MTL II und der Gemeinden - BMT-GII). Der 2005 abgeschlossene „Tarifvertrag für den öffentlichen Dienst“ - TVöD führt ein einheitliches Entgeltsystem für Arbeiter und Angestellte ein. 
- Demgegenüber erfolgt die Festlegung der Dienstverhältnisse der Beamten auf der Basis der ,hergebrachten Grundsätze des Berufsbeamtentums“ (Art. 33 Abs. 5 GG) ausschließlich durch Gesetze. ${ }^{2}$ Die Dachverbände der Beamten, DBB und DGB, sowie deren Mitgliedsverbände, die auf Basis der in Art. 9 Abs. 3 GG garantierten Koalitionsfreiheit bestehen, sind nach herrschender Rechtsprechung und -lehre weder zur Führung von Tarifverhandlungen berechtigt noch verfügen sie über das wichtigste kollektive Druckmittel, das Streikrecht.

Die Ausführungen beziehen sich im Wesentlichen auf den Tarifbereich mit dem Regelungsmechanismus Tarifvertrag, da Arbeitgeberverbände nur in diesem Teil des ÖD vorhanden sind. Wir analysieren neben den Mitgliederstrukturen jeweils auch die Interessenpolitik der Verbände und gehen (für die kommunale Ebene) sowohl auf die Mitgliedsverbände als auch auf den Dachverband ein.

Die Organisation der Arbeitgeber folgt strikt der rechtlich vorgegebenen und für den gesamten Aufbau eines föderalistischen Staates wesentlichen Unterscheidung in Bund, Länder und Gemeinden und damit letztlich dem Regionalprinzip der horizontalen Integration; das ansonsten geltende Fachprinzip der vertikalen Integration wird ausschließlich verbandsintern befolgt. Der „Staat“ hat eine Doppelfunktion, da er zugleich Arbeitgeber und damit an der kollektiven Aushandlung der Arbeitsbedingungen unmittelbar beteiligt ist.

\section{Die verbandliche Organisation der Kommunen}

\subsection{Kommunale Arbeitgeberverbände}

Auf der Ebene der Kommunen bestehen als rechtsfähige Vereine des privaten Rechts kommunale Arbeitgeberverbände (KAV) als Vereinigungen von Arbeitgebern im Sinne des Tarifvertragsgesetzes. Die Verbandsgrenzen sind mit denen der Bundesländer identisch. Die von der Beschäftigtenzahl (Arbeiter und Angestellte) her größten und dadurch vom Stimmanteil in der Spitzenorganisation, der Vereinigung der kommunalen Arbeitgeberverbände (VkA), her wichtigsten KAV sind die der größten Bundesländer (NordrheinWestfalen, Bayern, Baden-Württemberg, Niedersachsen).

Die KAV erfüllen ebenso wie ihr Dachverband ausschließlich Arbeitgeberaufgaben hinsichtlich der Angestellten und Arbeiter, nehmen jedoch nicht die Aufgaben der Kommunen als Dienstherren von Beamten wahr. Für diesen gut 177.000 Personen umfassenden und damit vergleichsweise kleinen Bereich ist der kommunale Spitzenverband zuständig, bei dem der jeweilige Dienstherr Mitglied ist.

Der Zweck der KAV besteht in der Wahrung der gemeinsamen Interessen ihrer Mitglieder als Arbeitgeber und im Interessenausgleich zwischen ihnen sowie mit ihren Arbeitnehmern. Diese Aufgaben erfüllen die KAV insbesondere durch den Abschluss von Tarifverträgen und durch Hilfe und Beratung für ihre Mitglieder in allen Fragen des Arbeits- und Tarifrechts sowie bei Rechtsstreitigkeiten vor den Arbeits-, Sozial- und Verwaltungsgerichten.

Die Mitgliedschaft einer KAV, die wegen der privatrechtlichen Basis freiwillig ist, können satzungsgemäß u. a. erwerben: Gemeinden; Gemeindeverbände; Zweckverbände 
und Verwaltungsgemeinschaften; öffentliche Spar- und Girokassen; Anstalten und Stiftungen des öffentlichen Rechts. Damit ist infolge einer großen organisatorischen Breite eine gewisse Heterogenität der Interessen vorgegeben, die durch innerverbandliche Vorkehrungen und Mechanismen reduziert bzw. vereinheitlicht werden muss.

Die für die interne Politikformulierung und -koordinierung zuständigen Organe eines KAV können laut Verbandssatzung sein: Mitgliederversammlung, Hauptausschuss, Vorstand und Gruppenversammlungen bzw. -ausschüsse. Hierbei können Unterschiede zwischen den KAV hinsichtlich der Zusammensetzung und Aufgaben der Organe bestehen. Statuarische und exekutive Funktionen werden stets deutlich voneinander getrennt.

Die Mitgliederversammlung setzt sich aus je einem Vertreter der Verbandsmitglieder oder den Mitgliedern der Gruppenversammlungen bzw. -ausschüsse plus Vorstand zusammen; sie wird vom Vorstand mindestens einmal pro Jahr einberufen. Das Stimmrecht kann nach der Anzahl der beschäftigten Arbeitnehmer gestuft sein. ${ }^{3}$ Derartige differenzierte Stimmrechte sind auch von Unternehmensverbänden der Privatwirtschaft bekannt, bei denen u. a. Unternehmensgröße, Beschäftigtenzahl und Umsatz als Kriterien herangezogen werden können (Rampelt 1979: 8 f.). Zum Aufgabenbereich gehören vor allem Beschlüsse der Regularien wie die Genehmigung des Haushaltsplans, die Abnahme der Jahresrechnung und Bestellung der Rechnungsprüfer, die Beschlussfassung über Änderungen der Satzung, aber auch die Wahl des Vorstands.

Die Mitgliederversammlung ist, u. a. aufgrund der langen Abstände zwischen ihren Tagungen und wegen ihrer Größe, nicht als das entscheidende Gremium verbandlicher Willensbildung anzusehen; sie dient eher der formaldemokratischen Legitimation der Verbandsführung als der faktischen Entscheidungsfindung bei aktuellen Problemen.

Der Haupt- oder Verhandlungsausschuss setzt sich im Wesentlichen aus dem Vorstand und den Vorsitzenden der Gruppenversammlungen bzw. -ausschüsse zusammen; er ist damit ein im Vergleich zur Mitgliederversammlung kleines Gremium. Dieser Ausschuss trifft die anstehenden tarifpolitischen Entscheidungen, vor allem diejenigen, die mehrere Verbandsgruppen betreffen und daher einheitliche Regelungen notwendig machen. Er hat insbesondere Tarifverträge vorzubereiten und abzuschließen sowie die spezifischen Interessen der Gruppen zu koordinieren und über deren Vorschläge zu entscheiden.

Der Hauptausschuss stellt aufgrund seiner Größe und satzungsrechtlichen Kompetenzen ein wichtiges Beschluss- und Lenkungsgremium dar. Die unterschiedlichen Interessen der Mitgliedergruppen werden bei der innerverbandlichen Willensbildung dadurch berücksichtigt bzw. eingebunden, dass neben dem Vorstand auch die Gruppenversammlungen bzw. -ausschüsse entweder durch Mitglieder oder durch ihren Vorsitzenden im Hauptausschuss vertreten sind. Durch diese institutionell abgesicherte Breite der Zusammensetzung wird das gesamte Interessenspektrum der Mitglieder in dieser „kleinen Mitgliederversammlung“" als handlungsfähigem Entscheidungsorgan repräsentiert. Der Hauptausschuss ist das entscheidende Koordinationsgremium für die Gruppenversammlungen bzw. -ausschüsse.

Der Vorstand wird von der Mitgliederversammlung oder dem Hauptausschuss auf Zeit, d.h. für vier Jahre, gewählt und soll bei einer geringen absoluten Größe repräsentativ

3 Typisch ist eine Regelung wie die folgende: 1-500 Arbeitnehmer eine Stimme, 501-1000 Arbeitnehmer zwei Stimmen, mit mehr als 1000 Arbeitnehmern für je angefangene 1000 Arbeitnehmer je eine zusätzliche Stimme. Solche Regelungen führen im Übrigen zu einer Vormachtstellung der großen Mitglieder (,Verbandsoligarchie"). 
hinsichtlich der Mitgliedergruppen zusammengesetzt sein, was deren Integration sowie den Interessenausgleich zwischen ihnen erleichtert. Der Vorstand erfüllt zum einen formale Aufgaben, u. a. die Wahl des Vorsitzenden, Entscheidung über Aufnahmeanträge, Vorbereitung und Einberufung der Mitgliederversammlung; zum anderen entscheidet er häufig in den übrigen Gremien mit, wobei die satzungsrechtlich abgesicherte Mitgliedschaft in verschiedenen Verbandsorganen ebenso hilfreich ist wie die repräsentative und daher austarierte Zusammensetzung.

KAV sind wie Arbeitgeberverbände der Privatwirtschaft „encompassing organizations" (Olson 1982) und von den Interessenlagen ihrer Mitglieder her keinesfalls homogene Gruppierungen. Das zentrale Problem besteht darin, dass die heterogenen Interessen, die innerhalb von sowie vor allem zwischen großen Mitgliedergruppen bestehen, mediatisiert und vereinheitlicht werden müssen, um kollektives Handeln zu ermöglichen bzw. um die externe Handlungsfähigkeit des Verbandes zu sichern. Innerhalb der KAV wählen deshalb die Gruppenversammlungen, d.h. die Versammlungen aller Mitglieder einer Gruppe, für die Dauer von vier Jahren die Mitglieder der sogenannten Gruppenausschüsse nach differenziertem Stimmrecht. Gruppenausschüsse, die eine horizontal-innerverbandliche Differenzierung der Willensbildung bezwecken bzw. bewirken, bestehen als ständige Ausschüsse u.a. für folgende Bereiche: Verwaltung (einschließlich Flughäfen), Sparkassen, Versorgungsbetriebe, Häfen, Nahverkehr, Kranken-, Heil- und Pflegeanstalten.

Ihre Anzahl und Aufgaben differieren geringfügig nach regional-länderspezifischen Besonderheiten sowie im Zeitablauf. Generell besteht neben einer grundsätzlich eng begrenzten Anzahl eine weitgehende inhaltliche Parallelität zu den noch zu behandelnden Gruppenausschüssen der Dachorganisation, dem „Verband der Verbände“, wodurch interne Komplikationen vermieden werden. Die funktionale Differenzierung der Gruppenausschüsse erlaubt eine arbeitsteilig-kooperative Strategie des Verbandes. ${ }^{4}$

Die Gruppenausschüsse bearbeiten und beraten die besonderen, vor allem tarifpolitischen Angelegenheiten ihrer Gruppenmitglieder bzw. ihres Fachgebiets und unterbreiten den Verbandsorganen Vorschläge; insofern erfüllen sie wichtige Zulieferer- und Vorbereitungsfunktionen. Häufig führen die Gruppenausschüsse in eigener Verantwortung die Tarifverhandlungen, die ausschließlich ihre Mitglieder betreffen. Sie verfügen sowohl satzungsrechtlich als auch tatsächlich über weitgehende Entscheidungskompetenz und -autonomie hinsichtlich der Behandlung von Problemen ihrer Verbandsgruppe, wobei sie als Randbedingung ihres Handelns das allgemeine Tarifgefüge wahren müssen. Die übrigen ,legislativen" Verbandsorgane, also der Hauptausschuss bzw. die Mitgliederversammlung, mit denen zwischenzeitlich Absprachen getroffen werden, legitimieren später die in den Gruppenausschüssen gefallenen Sachentscheidungen.

Im Vergleich zum Gesamtverband sind Gruppenausschüsse von der Interessenlage her relativ homogene Organe der Entscheidungsfindung und -absicherung, was Probleme der Interessenvereinheitlichung wesentlich mildert. Diese innerverbandliche Entscheidungsdezentralisierung in fachlich begrenzten Sachfragen hat mehrere Funktionen: Zum einen garantiert sie einen hohen Beteiligungsgrad der Mitglieder an der Willensbildung und erleichtert deren Integration durch die Verpflichtung auf gemeinsame Beschlüsse; zum anderen lässt sie potentielle Bewertungsunterschiede frühzeitig deutlich werden und ermöglicht die

4 KAV und deren Gruppenausschüsse können Gastmitglieder ohne Stimmrecht haben, etwa kommunale Spitzenverbände oder Unternehmen, bei denen die öffentliche Hand keine Majorität hat, wie bestimmte Krankenhäuser oder Lebenshilfeorganisationen. 
Konfliktbewältigung durch internes Interessenclearing. Die langjährigen Erfahrungen mit dieser Organisationsstruktur werden von den Verbandsvertretern durchweg sehr positiv beurteilt (Keller 1987a).

Personelle und institutionelle Verflechtungen von Verbandsgremien in horizontaler und vertikaler Richtung erleichtern die Formulierung eines einheitlichen Verbandswillens durch Integration der Teilentscheidungen. Eine ursprünglich auf die Arbeitgeberverbände der Privatwirtschaft gemünzte Aussage gilt auch für die des ÖD: „Durch die Differenzierung der Verbandsorganisation in Subsysteme, deren Bezugsrahmen sich an mitgliederrelevanten Interessendimensionen orientiert (z.B. Fachgruppen), wird deren zeitlich, sachlich und sozial generalisierte Einbeziehung in den Ablauf der Politikformulierung gewährleistet." (Traxler 1980: 8).

Abstimmungen sind nach übereinstimmender Auskunft in der Regel nicht kontrovers. Diese Angaben decken sich mit der allgemeinen Erkenntnis (Rampelt 1979: 59f.), dass bei Verbänden mit freiwilliger Mitgliedschaft Kampfabstimmungen ebenso selten sind wie Fraktionsbildungen, u. a. weil solche Strategien solidarisches Handeln kaum gewährleisten könnten. Stattdessen setzen sie auf „Konsensbildung als Vereinheitlichungsstrategie“ (Traxler 1985: 62). ${ }^{5}$ Formale Abstimmungen ratifizieren häufig nur bereits auf konsensualer Basis gefallene Entscheidungen.

\subsection{Der Dachverband}

Die rechtlich selbständigen KAV haben sich zu einer Spitzenvereinigung im Sinne des Tarifvertragsgesetzes zusammengeschlossen, der Vereinigung der kommunalen Arbeitgeberverbände (VKA). Mitglieder können nur Verbände, nicht hingegen einzelne Arbeitgeber über direkte Mitgliedschaft werden; ähnlich verläuft die vertikale Integration bei den „Verbänden der Verbände" in der Privatwirtschaft.

Der tarifpolitische und arbeitsrechtliche Dachverband VKA verfolgt den Zweck, als Spitzenvereinigung

„die gemeinsamen Angelegenheiten ihrer Mitglieder und der diesen angeschlossenen Arbeitgeber auf tarif-, arbeits- und sozialrechtlichem Gebiet gegenüber Gewerkschaften, staatlichen Stellen und anderen Organisationen zu vertreten, insbesondere hat sie

1. die Grundsätze der Tarifpolitik festzulegen,

2. Tarifverträge abzuschließen,

3. verbindliche Richtlinien festzulegen oder zu vereinbaren,

4. für den Abschluss von Tarifverträgen durch die Mitglieder verbindliche Grundsätze festzulegen oder zu vereinbaren,

5. den Erfahrungsaustausch zwischen den Mitgliedern zu vermitteln“(VKA 2003: §2).

Die VKA erfüllt wesentliche Koordinationsaufgaben; sie befasst sich mit allen Fragen von allgemein-überregionalem Interesse. Die für die interne Willensbildung zuständigen Organe sind Mitgliederversammlung, Präsidium und Vorstand.

5 Ähnlich bei einem internationalen Vergleich: „,...) there is universally strong preference for consensual decision-making in association affairs and a correspondingly strong disinclination to let internal differences be exposed to public view or to the scrunity of union strategies." (Windmuller 1984: 14). 
Die Mitgliederversammlung als oberstes Organ tagt satzungsrechtlich jährlich mindestens einmal, tatsächlich jedoch häufiger, vor allem während der Tarifverhandlungen. Ihre Aufgaben bestehen vor allem in grundlegenden Entscheidungen, insbesondere dem Abschluss und der Kündigung von Tarifverträgen. Die Mitgliederversammlung besteht aus den Vertretern der Mitgliedsverbände, deren Stimmanteil nach ihrer Größe gestaffelt wird, was zu einer Vormachtstellung der Verbände aus den bevölkerungsstarken Bundesländern führt. Bei der internen Willensbildung wird auf breite Zustimmung Wert gelegt, die durch intensive „Überzeugungsarbeit“ erreicht wird und die innerverbandliche Durchsetzung von Beschlüssen erleichtert. ${ }^{6}$

Das engere, für tarifpolitische Entscheidungen maßgebende Gremium ist das Präsidium, das sich aus den Vorsitzenden der Mitgliedsverbände, den Vorsitzenden der Gruppenausschüsse und dem Hauptgeschäftsführer zusammensetzt. Die laufenden Geschäfte führt der Vorstand.

Die Mitglieder der VKA sind ebenso wie die der Tarifgemeinschaft deutscher Länder (vgl. Abschnitt 3) nur in begrenztem Umfang berechtigt, selbständige Tarifverträge oder sonstige arbeitsrechtliche Vereinbarungen abzuschließen. Sie treten aufgrund formaler Regelungen in den Verbandssatzungen (VKA 2003: § 6; TdL 2005: § 5) mit dem Erwerb der Verbandsmitgliedschaft wesentliche Rechte aus dem Kanon ihrer Tarifhoheit für die Fälle $\mathrm{ab}$, in denen die Spitzenorganisation Verträge abschließt oder sich den Abschluss vorbehält (sogenannter Verbandszwang). Ähnlich ist die Situation in der Privatwirtschaft (Bunn 1984: 175).

Im Binnenverhältnis zwischen Mitgliedsverbänden und Spitzenorganisation hat in langfristiger Perspektive ähnlich wie bei anderen Arbeitgeberverbänden ${ }^{7}$ eine innerorganisatorische Zentralisierung der Entscheidungskompetenzen stattgefunden: Der Dachverband schließt die wesentlichen, d.h. bundesweit geltenden Tarifverträge ab; er wahrt und sichert faktisch die Einheitlichkeit der Tarifpolitik durch Kontrolle der tarifpolitischen Willensbildung, obwohl er formal keinerlei Weisungsbefugnisse gegenüber den Mitgliedsverbänden hat (Erhöhung der Effektivität der Verhandlungen).

Gruppenausschüsse sind nicht nur bei den Mitgliedsverbänden, sondern auch beim Dachverband eingerichtet. Gemäß VKA-Satzung ( $§ 16)$ bestehen folgende Ausschüsse: Flughäfen, Krankenhäuser und Pflegeeinrichtungen, Nahverkehrsbetriebe und Häfen, Sparkassen, Verwaltung, Versorgungsbetriebe (zu Einzelheiten http:/www.vka.de/vka/home/fachbereiche.shtml). In diese von der Mitgliederversammlung eingerichteten Gruppenausschüsse entsendet jeder Mitgliedsverband ein ordentliches sowie ein stellvertretendes Mitglied; in der Regel sind dies die Vorsitzenden und stellvertretenden Vorsitzenden der Gruppenausschüsse der einzelnen KAV. Durch eine sorgsame Austarierung der personellen Zusammensetzung wird eine Repräsentation aller größenbedingten, regionalen, gruppenspezifischen und sonstigen Partikularinteressen im jeweiligen Gruppenausschuss der Spitzenorganisation erreicht sowie die Verpflichtungsfähigkeit der Mitglieder (Weitbrecht 1969) erhöht. Die im Vergleich

6 Aufgaben der Mitgliederversammlung sind u. a.: die Wahl des Vorsitzenden der VKA und dessen Stellvertreters, die Bildung und Auflösung der Gruppenausschüsse, der Beschluss über den Abschluss und die Kündigung von Tarifverträgen, Beschlüsse über Richtlinien.

7 Allgemein gilt: „Decision-making in employers associations is generally a centralized matter, rather removed from the reach of individual member firms except for local or regional associations. Insofar as this situation indicates a concentration of authority in relatively small boards and in top association officials, it reflects the fact that participatory democracy as a form of internal government is not characteristic of employers associations.“(Windmuller 1984: 16). 
zu anderen Gremien geringe Größe verstärkt die Handlungs- und Entscheidungsfähigkeit im Rahmen einer flexibel gehaltenen Arbeitsteilung.

Die Gruppenausschüsse sind auch auf VKA-Ebene Fachausschüsse, die die inhaltliche Verbandsarbeit faktisch weitgehend leisten und infolge ihrer Besetzung über „Sachverstand" und „Kompetenz" verfügen. Die auch auf dieser Ebene dezentralisierten Befugnisse sind recht weitgehend. ${ }^{8}$ Diese Vertretungen der Fachinteressen sind aufgrund ihrer relativen Autonomie als funktionale Äquivalente zu „Fachverbänden“ anzusehen. Sie werden bei Verhandlungen, die ihren Zuständigkeitsbereich betreffen, einbezogen und erteilen fachlichen Rat. Die Häufigkeit der Zusammenkünfte richtet sich nach dem Arbeitsanfall; in der Regel tagen die Ausschüsse ein- bis zweimal jährlich.

Die Mitarbeit erfolgt ehrenamtlich. Dadurch wird die Verpflichtungsfähigkeit der Gruppenmitglieder erhöht, was in Anbetracht der Wichtigkeit der Beschlüsse notwendig ist: Die Gruppenausschüsse fällen de facto Entscheidungen, die innerverbandlich umgesetzt werden müssen. Durch die Mitarbeit in den Gruppenausschüssen treten keine Probleme auf. Schwierigkeiten wegen der zusätzlichen zeitlichen Belastung durch intensive Mitarbeit im Verband sind in der Privatwirtschaft größer, wo „die firmeninterne Tätigkeit allemal höhere Priorität als die Mitarbeit im Verband“ (Traxler 1985: 62; ähnlich Windmuller 1984: 14) besitzt. Im ÖD wird die Tätigkeit in Verbandsgremien als integrierter Teil der übernommenen Aufgaben definiert, zumal das Problem der Vertretung gelöst werden kann.

Eine wiederholte Entsendung von Mitgliedern ist bei der Mehrzahl der Ausschüsse die Regel; häufig sind Alters- oder Gesundheitsgründe für das Ausscheiden verantwortlich. Das Verhältnis zwischen hauptberuflichen Verbandsfunktionären, der Geschäftsführung und ehrenamtlichen Repräsentanten der Mitglieder wird von Ersteren als in der Regel konfliktfrei beschrieben. ${ }^{9}$ Innerhalb solcher „dualistic leadership structures“ (Schmitter/Streeck 1981: 54) erschwert die geringe Zahl hauptberuflicher Mitarbeiter eine weitgehende Loslösung der Verbandsspitze bzw. der von ihr betriebenen Politik von den Interessen der Mitglieder; auch das Ausmaß der Entscheidungsautonomie sollte nicht überschätzt werden.

\subsection{Verbandsressourcen und -probleme}

Auf kommunaler Ebene werden die Mittel für die Aufwendungen der Verbände ausschließlich durch Beiträge der Mitglieder aufgebracht. Bemessungsgrundlage bei den KAV ist die Zahl der Arbeitnehmer ihrer Mitglieder; derartige Regelungen sind auch in der Privatwirtschaft üblich (Rampelt 1979: 37-42). Im Übrigen implizieren unterschiedliche Beiträge häufig differenzierte Stimmrechte.

Die Beitragsehrlichkeit stellt in der Privatwirtschaft vor allem bei kleineren Unternehmen, im ÖD dagegen kaum ein Problem dar, da in Letzterem die Bemessungsgrundlage

8 Die Gruppenausschüsse ,haben die ihr Fachgebiet betreffenden Angelegenheiten zu beraten. Soweit nicht ein Organ der VKA zuständig ist, können sie bindende Beschlüsse fassen, um für ihren Bereich die Einheitlichkeit der Arbeitsbedingungen zu sichern" (VKA-Satzung § 16).

9 Für die Arbeitgeberverbände der Privatwirtschaft gilt hinsichtlich dieses Verhältnisses: „Employers associations (...) combine in their decision processes direct participation by both professional staff and managerial representatives, the latter drawn broadly from members firms, reflecting not only the operational requirement that the diversity among firms be allowed to express itself in the decision processes but also the reality that consensus and compromise have to be recurringly sought among member firms within the employers associations (...)“ (Bunn 1984: 191). 
„objektiv“ festzustellen ist. Die jährlichen Mitgliedsbeiträge der KAV bilden die ausschließliche Finanzierungsgrundlage der VKA, wobei auch hier doppelt nach der Verbandsgröße differenziert wird: Der Beitrag setzt sich aus einem Grund- und einem Zusatzbeitrag zusammen, die beide nach der Beschäftigtenzahl berechnet werden (VKA-Satzung $\S 8)$. Insgesamt ist die Mitgliedschaft sowohl in absoluten Beträgen als auch im Verhältnis zu der bei anderen Verbänden verhältnismäßig günstig.

Die personelle Ausstattung der KAV ist nicht besonders umfangreich. Die KAV haben in aller Regel wesentlich weniger als zehn Mitarbeiter. Dieser Sachverhalt, der geringe Ausgaben des Verbandes zur Folge hat, kann erklärt werden durch die interne „Straffung der Verbandsarbeit", durch das erhebliche Ausmaß ehrenamtlicher Mitarbeit, u.a. in den Gruppenausschüssen, sowie durch den geringen Umfang der Öffentlichkeitsarbeit.

\section{Die Organisation der Länder}

Die Bundesländer haben sich zu einer eigenständigen Arbeitgebervereinigung im Sinne des Tarifvertragsgesetzes zusammengeschlossen, der Tarifgemeinschaft deutscher Länder (TdL), die wie die VKA seit 1949 besteht. ${ }^{10}$ Ziel ist die „Wahrung der Interessen ihrer Mitglieder an der Einheitlichkeit der Arbeitsbedingungen des öffentlichen Dienstes“. Die TdL verfolgt dieses bewusst flexibel gehaltene Ziel vor allem durch den ihr im Wesentlichen vorbehaltenen „Abschluss von Tarifverträgen und sonstigen Vereinbarungen“ für ihre Mitglieder. Ihre Organe sind die Mitgliederversammlung, der Vorstand und der Vorsitzende des Vorstandes.

Vertreter der TdL nach außen und damit auch Repräsentant der Länder bei Tarifverhandlungen ist der auf Vorschlag der Finanzministerkonferenz gewählte Vorsitzende des dreiköpfigen Vorstands. ${ }^{11}$ Traditionell ist das Finanzministerium bei der Mehrzahl der Länder das für das Tarifwesen zuständige Ressort. ${ }^{12}$ Nach einer allgemein akzeptierten Regel soll der für das Dienstrecht der Beamten zuständige Minister zugleich auch für das Tarifwesen verantwortlich sein (Konzentration der Kompetenzen).

Die TdL finanziert sich wie die KAV und die VKA ausschließlich über die Beiträge ihrer Mitglieder, wobei alle Länder denselben Betrag zahlen und gemäß Satzung auch über das gleiche Stimmrecht in der Mitgliederversammlung verfügen. Die Tatsache, dass es im Gegensatz zur VKA kein differenziertes Stimmrecht gibt, schließt allerdings nicht aus, dass die größeren Bundesländer im Meinungsbildungsprozess über größeres Gewicht verfügen können. Die Mitgliederversammlung beschließt mit einer qualifizierten Mehrheit von drei Fünfteln der abgegebenen Stimmen. Zu ihrem Aufgabenbereich gehört neben einigen Regularien die Wahl und Abberufung der Vorstandsmitglieder und vor allem die „Beschluss-

10 „The meeting of April 22, 1949, at which the TDL was founded was attended by representatives of six state associations of local governments. They decided, however, that they would set up their own national federation rather than join with the complexity of their organizations, it appears that this was a wise decision." (McPherson 1971: 47).

11 Folgende Regelung ist zu beachten: „Vorstandsmitglieder können nur Vertreter der Mitglieder sein, wenn zum Geschäftsbereich des Vertreters nach der Geschäftsordnung der Landesregierung die Zuständigkeit für das Tarifrecht der Arbeitnehmer des Landes gehört.“ (TdL-Satzung § 11).

12 Besonderheiten wie die Zuordnung zum Innenministerium in Hessen und dem Saarland oder zu bestimmten Senatskommissionen in den Stadtstaaten sind durch die historische Entwicklung der jeweiligen Geschäftsverteilungspläne zu erklären. 
fassung über Maßnahmen zur Sicherung der Einheitlichkeit der Arbeitsbedingungen der Arbeitnehmer der Länder, insbesondere über Abschluss und Kündigung von Tarifverträgen und sonstigen Vereinbarungen" (TdL-Satzung $§ 10$ ). Die Mitgliederversammlung verfügt damit über vergleichsweise weitreichende Kompetenzen.

Formale Beschlüsse der Mitgliederversammlung, die während der Verhandlungen als Beschlussorgan agiert, kommen überwiegend einvernehmlich zustande. Besonders bei Fragen von grundsätzlicher Bedeutung werden breite Mehrheiten angestrebt. Sogenannte Kampfabstimmungen finden so gut wie nie statt; Gegenstimmen sind selten. In besonderen Situationen, etwa bei Nichtbetroffenheit, kommen gelegentlich Stimmenthaltungen vor (Keller 1987b). Diese Verhaltensstrategien decken sich mit denen anderer Arbeitgeberverbände.

Die Meinungsbildung der TdL verläuft wegen der geringen Mitgliederzahl straffer und zügiger als bei den KAV: Zunächst werden bei den Ländern die Vorstellungen innerhalb der zuständigen (Finanz-)Ministerien koordiniert, wobei die Innenministerien einbezogen werden. Die Länder stehen über ihre zuständigen Ministerien in permanentem Kontakt, der vor allem über die Verbandsgeschäftsstelle vermittelt wird. Nach Bekanntgabe bzw. Übermittlung der gewerkschaftlichen Forderungen werden intern Kostenrechnungen angestellt, Argumente gesammelt und Verhandlungsstrategien festgelegt. Die auf Länderebene zuständigen Minister, Staatssekretäre und Referenten leiten in Vorgesprächen Abstimmungsprozesse unter den Beteiligten ein.

Auf der Ebene der Länder bestehen keine Gruppenausschüsse wie bei KAV und VKA. Die TdL bildet jedoch Verhandlungskommissionen für besondere Bereiche, wie Finanz- oder Justizverwaltung, ${ }^{13}$ die aufgrund der gesetzlich vorgegebenen Aufgabenverteilung zwischen Bund, Ländern und Kommunen in die Zuständigkeit der Länder fallen. Die kompetenten und interessierten Mitglieder sind in den jeweiligen Verhandlungskommissionen vertreten. Im Übrigen beteiligt sich die TdL informell an den Gruppenausschüssen der VKA, die für den Bereich der Länder ebenfalls relevant sind, z. B. Krankenanstalten, Nahverkehr; hier spielen Aspekte der Informationsgewinnung eine Rolle, z. B. im Nahverkehr.

\section{Die Willensbildung auf Bundesebene}

Auf der Ebene des Bundes gibt es im Gegensatz zu den Kommunen und Ländern keine verbandliche Organisation. Einige Probleme, die in Arbeitgeberverbänden auftreten und gelöst werden müssen, erfordern hier eine Koordination zwischen Ministerien. ${ }^{14}$

Die Federführung liegt wegen seiner Zuständigkeit für alle besoldungsrechtlichen und tarifvertraglichen Regelungen der Beschäftigungsbedingungen beim BMI; bei Verhandlungen gilt das „Prinzip des Einvernehmens“ mit dem BMF.

13 Weiterhin bildet die TdL bei Bedarf Ausschüsse (Forstfragen), Kommissionen (Zusatzversorgung, Lehrerkommission, Satzungsangelegenheiten, Haushalt) und Projektgruppen (Wissenschaft), die Probleme analysieren und Lösungsvorschläge unterbreiten, aber keine formalen Entscheidungsbefugnisse haben.

14 Bis 1960 führte der Finanzminister (BMF) die Verhandlungen; seitdem beauftragt die Bundesregierung den Bundesminister des Innern (BMI) förmlich mit der Interessenwahrnehmung. „This responsibility (...) was transferred because there was a widespread belief that the Finance Ministry was too exclusively concerned with the goal of minimizing expenditure. Finance continues, however, to have a representative present as observer in all federal negotiations." (McPherson 1971: $45 \mathrm{f}$.). 
„Die Organisation auf Arbeitgeberseite ist durch die Trennung der Verhandlungsverantwortung (Innenministerium) und Finanzverantwortung (Finanzministerium) zu einer größeren Effektivität der Verhandlungen gekommen, weil mehr Möglichkeiten des stufenweisen Herantastens an das Limit notwendig sind und damit Verhandlungen entstehen, die den innerorganisatorischen Anpassungsprozessen eher Rechnung tragen"(Weitbrecht 1973: 16).

Die institutionelle Grundlage dieser engen und laufenden Zusammenarbeit stellt die Bundeshaushaltsordnung dar, die Personalausgaben nur auf gesetzlicher und tarifvertraglicher Basis vorsieht.

Die unterschiedlichen Vorstellungen hinsichtlich des Verhandlungsspielraums und der tarifpolitischen Strategie werden in einem nichtformalisierten Verfahren intern abgestimmt mit den an den jeweiligen Verhandlungen beteiligten anderen Instanzen (vor allem Bundeskanzleramt, Finanz- und Wirtschaftsministerium). ${ }^{15}$ Bei dieser Willensbildung sind unterschiedliche Bewertungen am ehesten aus dem Wirtschafts- und besonders dem Finanzministerium zu erwarten, so dass eine Koordination bzw. ein Ausgleich spezieller Interessen angestrebt werden muss. Dabei treten parteipolitische Überlegungen zugunsten tarifpolitischer Sachpositionen in den Hintergrund: Alle Beteiligten respektieren die Autonomie der Tarifverhandlungen. Kabinettsinterne Konflikte etwa der Art, dass bei Koalitionsregierungen die beteiligten Ministerien von Politikern verschiedener Parteien besetzt sind und diese versuchen, das Wählerreservoir in ihrer eigenen Partei zu vergrößern, treten nicht auf.

Der BMI ist nicht nur für die Erfüllung der Arbeitgeberaufgaben zuständig; im Rahmen der konkurrierenden Gesetzgebung des Bundes, die vollständig ausgenutzt wird, bereitet er durch die Unterabteilung Besoldungsrecht der Dienstrechtsabteilung seines Ministeriums auch die Gesetzesentwürfe der Bundesregierung zur Besoldungsanpassung der Einkommen aller Beamten verantwortlich vor. ${ }^{16}$ Damit verfügt der BMI über die zentrale Position bei der Festsetzung der Einkommen aller öffentlich Bediensteten; er ist als zentrale Koordinationsinstanz für besoldungsrechtliche und tarifvertragliche Regelungen und als Träger der gesamten staatlichen Einkommenspolitik anzusehen. Diese Zentralisierung der Kompetenzen erleichtert die notwendigen komplexen Koordinations- und Abstimmungsprozesse, die langfristig das Ziel einer Angleichung der materiellen Arbeitsbedingungen im Tarif- und Besoldungsbereich verfolgen.

Neben der Aufgabe, am Abschluss von Tarifverträgen mitzuwirken, existieren auch auf der Ebene des Bundes weitere Aufgaben ,unterhalb“von Tarifverhandlungen: Ähnlich wie bei den übrigen Arbeitgebern gehören arbeitsrechtliche Angelegenheiten dazu, wobei aber keine Prozessvertretungen übernommen werden; allerdings ist der Beratungsbedarf bei Verbänden größer und stärker ausgeprägt als im Verkehr zwischen Ministerien. Wichtiger sind Aufgaben wie die Beobachtung aktueller Entwicklungen in der Rechtsprechung oder die Ausarbeitung von Richtlinien für die Anwendung tarifrechtlicher Regelungen. Hier nimmt die Unterabteilung Tarifrecht der Dienstrechtsabteilung eine Zentralfunktion für den gesamten Bundesbereich wahr. ${ }^{17}$

15 An diesem Abstimmungsprozess können je nach Gegenstand der Verhandlungen auch andere Ministerien beteiligt sein: Bei personengruppen- und bereichsspezifischen Verhandlungen sind etwa die personalstarken Ressorts eingebunden, z. B. das Bundesministerium der Verteidigung wegen der Zivilbediensteten; an anderen Verhandlungen ist etwa das Auswärtige Amt beteiligt.

16 Diese Konstellation der Befugnisse wird sich vor allem infolge der Föderalismusreform ändern.

17 Hinzu kommen bestimmte Serviceleistungen für Anstalten und Stiftungen des öffentlichen Rechts: So sind etwa die bundesunmittelbaren Sozialversicherungsträger wie die Bundesversicherungsanstalt für Angestellte 


\section{Erklärungen}

\subsection{Privatwirtschaft und ÖD}

Eigenständige theoretische Erklärungen zu den Verbänden der Arbeitgeber des ÖD liegen nicht vor. Die wenigen vorhandenen, auf die Privatwirtschaft gerichteten Beiträge sind aufgrund der unterschiedlichen Rahmenbedingungen nur begrenzt und ausschließlich für die Verbände der kommunalen Ebene anwendbar.

Die KAV weisen in wichtigen, vor allem organisationsstrukturellen Dimensionen gewisse Parallelen zu Arbeitgeberverbänden der Privatwirtschaft auf (u. a. Integration und Mediatisierung heterogener Interessen, Verbandsressourcen, Erstellung privater Dienstleistungen). ${ }^{18}$ Ein wesentlicher Unterschied besteht darin, dass die in Verbänden organisierten Arbeitgeber des ÖD weder auf Produktmärkten noch auf Arbeitsmärkten in einem internen oder externen Konkurrenzverhältnis, der Erfolgsbedingung privater Akkumulation, zueinander stehen: Die Produktmärkte sind doppelt differenziert, horizontal durch lokal bzw. regional begrenzte Zuständigkeiten, vertikal durch die zwischen den Gebietskörperschaften auf der Basis grundgesetzlich vorgegebener Regelungen bestehenden Kompetenzabgrenzungen. ${ }^{19}$ Die Arbeitsmärkte (Henneberger 1997, 2004) sind zum einen in der gegenwärtigen, regionalen und allgemeinen Beschäftigungssituation durch ein deutliches Überangebot gekennzeichnet, welches die bei den gegenteiligen Ausgangsbedingungen gegebene Konkurrenz um Arbeitskräfte weitgehend verhindert; zum anderen sind die Leistungen der verschiedenen Arbeitgeber seit den frühen 1970er Jahren weitgehend vereinheitlicht. $^{20}$

Wenn die Einzelinteressen nicht primär über den Markt realisiert werden, ist die Organisation im Verband nicht mehr von nur nachrangiger Bedeutung wie in der Privatwirtschaft. Unsolidarisches Handeln infolge dominierender Partikularinteressen bzw. autonomer unternehmerischer Individualentscheidungen im Rahmen einer profitorientierten Konkurrenzwirtschaft ist daher kaum zu erwarten. Das „Spannungsverhältnis zwischen individueller und organisierter Interessenverfolgung“ (Rampelt 1979: 1f.) ist weniger ausgeprägt; die Alternative, Resultate des kollektiven Verbandshandelns durch Einzelentscheidungen der Unternehmung zu verändern, hat kaum Bedeutung. Weiterhin sind auch Interessenunterschiede zwischen kleinen und großen Mitgliedern weniger relevant als in den Branchen der Privatwirtschaft; zudem bestehen keine Mittelstandsprobleme, so dass Vereinheitlichungsproble-

und die Bundesagentur für Arbeit tarifautonom und führen formal eigenständige Tarifverhandlungen; sie werden jedoch vom Bund an dessen Verhandlungen informell beteiligt. Weiterhin sind bestimmte Zuwendungsempfänger des Bundes nicht tarifgebunden, wenden aber das Tarifrecht an; so gibt es etwa Haustarifverträge bei der Kernforschungsanlage Jülich, die sich entsprechend orientieren.

18 Aus Studien über Arbeitgeberverbände der Privatwirtschaft wissen wir, dass diese neben ihrer Tariffunktion weitere Vertretungsaufgaben übernehmen. „Dazu zählen die Einflussnahme auf die Formulierung der Arbeits- und Sozialgesetzgebung und die Mitwirkung an ihrer Vollziehung im Rahmen der Arbeits- und Sozialgerichtsbarkeit sowie in den Selbstverwaltungsorganen der Sozialversicherung." (Traxler 1985: 55). Ähnliches gilt auch für die Arbeitgeberverbände des ÖD, die u.a. ehrenamtliche Arbeitsrichter (Arbeitgeberbeisitzer) bei den Arbeitsgerichten, den Landesarbeitsgerichten und dem Bundesarbeitsgericht sowie Sozialrichter bei den Sozial- und Landessozialgerichten stellen.

19 Wir abstrahieren hierbei von seltenen Ausnahmesituationen, die in Grenzgebieten von Bundesländern oder zwischen Großstädten und ihrem Umland (etwa bei Schulen) auftreten können.

20 Gewisse Konkurrenzbeziehungen bestehen auf kommunaler Ebene durch die Konkurrenz privater Anbieter in einzelnen Bereichen (u. a. Entsorgung, Nahverkehr). 
me für die Verbandspolitik leichter zu lösen sind. Schließlich brauchen auch unterschiedliche konjunkturelle Situationen innerhalb der Solidargemeinschaft kaum berücksichtigt zu werden. Last, not least ist auch das Verhältnis zwischen Arbeitgebern und Staat im ÖD anders strukturiert als in der Privatwirtschaft.

Formal existieren in der Bundesrepublik ähnlich wie in der Mehrzahl vergleichbarer Länder getrennte und voneinander unabhängige Verbände der privaten und öffentlichen Arbeitgeber. ${ }^{21}$ Der ÖD ist der einzige zentrale Sektor, dessen Arbeitgeber nicht in der Bundesvereinigung der Deutschen Arbeitgeberverbände (BDA) organisiert sind: Die öffentlichen Arbeitgeber sehen keine Veranlassung, sich der Verbandsdisziplin zu unterwerfen, die privaten Arbeitgeber haben Vorbehalte wegen der parteipolitischen Bindung und Durchdringung der Interessenvertretung innerhalb des ÖD. ${ }^{22}$

Seit Langem bestehen jedoch mehr oder weniger enge, in unregelmäßigen Abständen stattfindende Kontakte der Arbeitgeberverbände des ÖD zur BDA sowie zu den Mitgliedsverbänden; diese Kontakte finden vor allem auf der Geschäftsführerebene statt. Weiterhin dienen informelle Spitzengespräche der Verhaltensabstimmung und verfolgen u. a. das Ziel, Präjudizierungen zu vermeiden, die vor allem während der Tarifverhandlungen auftreten können. Die Existenz dieser nicht institutionalisierten Kontakte ist nicht verwunderlich in Anbetracht gewisser parallel gelagerter Interessen aller Arbeitgeber, etwa in Bezug auf Tarifverhandlungen, Entwicklungstendenzen des Arbeitsrechts oder Fragen der Mitbestimmung. Ein „Gedankenaustausch“ über wirtschafts- und sozialpolitische Fragen liegt im Eigeninteresse aller Beteiligten. Die Kontakte werden auch als förderlich für die Willensbildung im eigenen Verband angesehen.

Internationale Vergleiche zeigen, dass in einigen Ländern für die Privatwirtschaft die organisatorische Alternative ,einheitlicher Unternehmensverband vs. besonderer Arbeitgeberverband" relevant ist (Behrens/Traxler 2004). Als Merkmal der Organisation unternehmerischer Interessen in der Bundesrepublik gilt die häufig anzutreffende funktionale Differenzierung von Unternehmensverbänden in allgemeine Wirtschafts- und spezielle Arbeitgeberverbände, die Produktmarkt- bzw. Arbeitsmarktinteressen wahrnehmen. ${ }^{23}$ Hinsichtlich der Vertretung der gemeinsamen wirtschaftspolitischen Belange (u. a. Steuerwesen, Wirtschaftsrecht) gegenüber anderen Gruppen, Öffentlichkeit und Staat besteht im ÖD kein unmittelbares Pendant zu den entsprechenden Fachverbänden der Privatwirtschaft, insbesondere nicht zum BDI als wirtschaftspolitischer Säule des unternehmerischen Verbandswesens. Auf kommunaler Ebene weisen allerdings die kommunalen Spitzenverbände, d. h. der Deutsche Städtetag, der Deutsche Städte- und Gemeindebund sowie der Deutsche Landkreistag, deutliche Züge eines funktionalen Äquivalents auf. ${ }^{24}$ Sie bilden die Bundes-

21 „The general state of affairs can be summarized in the following terms (...) where government bodies have formed their own associations to negotiate with unions in the public services at national, regional, and local levels, these associations do not as a rule seek membership in essentially private federations of employers associations, nor are they likely to be admitted." (Windmuller 1984: 12).

22 „Basically the question is whether institutions created for the defence of private employer interests can admit public corporations into membership without diluting their essence. In most instances employers' associations have answered the questions in the negative, but there are many exceptions." (Windmuller 1987: 42, ähnlich 152).

23 Die für die Privatwirtschaft typische Doppelorganisation in fachliche und überfachliche Arbeitgeberverbände hat aufgrund der skizzierten Besonderheiten des ÖD keine unmittelbare Entsprechung.

24 Auf der Ebene der Länder besteht kaum die Notwendigkeit einer gesonderten Vertretung, da die Länder unmittelbar über den Bundesrat in das Gesetzgebungsverfahren eingebunden sind. 
vereinigung der kommunalen Spitzenverbände, die durchaus auch gemeinsam mit der VKA die Außenvertretung übernimmt und Interessenpolitik betreibt. ${ }^{25}$

Die Kontakte zu den kommunalen Spitzenverbänden sind enger und wichtiger als die zu den Arbeitgeberverbänden der Privatwirtschaft: Alle drei Verbände entsenden je einen Vertreter mit beratender Stimme in die Mitgliederversammlung der VKA. Außerdem schickt die Bundesvereinigung einen Vertreter mit beratender Stimme ins Präsidium der VKA. Derartige, von den Satzungen her gewollte und institutionell abgesicherte Verquickungen von Verbänden über die personelle Zusammensetzung der Gremien sind auch in den Spitzen- und Dachverbänden der Privatwirtschaft zu finden. Darüber hinaus bestehen zwischen VKA und Städtetag rege informelle Kontakte.

$\mathrm{Da}$ die Sonderinteressen der Mitglieder arbeitsteilig-kooperativ durch spezialisierte Verbände vertreten werden, besteht aus der Mikroperspektive ähnlich wie in der Privatwirtschaft Grund zu Doppel- und Mehrfachmitgliedschaften; diese sind wegen der Differenzierung der Interessenvertretung durch die verschiedenen Verbände unproblematisch und sinnvoll.

Insgesamt liegen die Organisationsgrade, d.h. die Anteile der verbandlich organisierten an allen Arbeitgebern, im ÖD höher als in der Privatwirtschaft. ${ }^{26}$ Für die kommunale Ebene gilt, dass der Organisationsgrad ,traditionell mit 80 bis 90 Prozent sehr hoch ist und auch die wenigen Nicht-Mitglieder die Verbandstarifverträge anwenden“ (Rosdücher 1994: 417). Auf der Ebene der Länder ist der Organisationsgrad durch den Ausschluss Berlins sowie durch den Austritt Hessens (vgl. Abschnitt 6) gesunken, liegt aber immer noch sehr hoch, da alle anderen Länder - trotz einiger wiederholter Austrittsdrohungen - bis dato Mitglieder der TdL geblieben sind. Bei einer Operationalisierung des Organisationsgrades über die Zahl der Beschäftigten anstatt der öffentlichen Arbeitgeber ändert sich an diesem Sachverhalt nichts. Schwierigkeiten mit den wenigen nichtorganisierten Arbeitgebern (etwa Versorgungs- oder Energiebetriebe, kommunale Wohnungsbaugesellschaften) sind nicht bekannt. Probleme der Entwicklung von Strategien zur Rekrutierung neuer Mitglieder stellen sich im Gegensatz zu Verbänden der Privatwirtschaft nicht; auch die Sicherung der Loyalität von Mitgliedern gelingt in aller Regel.

OT-Verbände, die eine Anpassung an veränderte Umweltbedingungen ermöglichen sollen, offerieren bekanntlich eine Verbandsmitgliedschaft mit allen üblichen Rechten und Pflichten, aber ohne Tarifbindung. Dieser Mitgliedschafts- bzw. Verbandstypus, der seit den mittleren 1990er Jahren in etlichen Branchen der Privatwirtschaft offensichtlich an Bedeutung gewinnt, ist im ÖD unbekannt; eine Entkoppelung von Tarif- und Dienstleistungsfunktion im Sinne eines Transformationsprozesses von Verbänden hat nicht stattgefunden.

\subsection{Die Theorie von Olson}

Spezifische Ansätze zur Erklärung von Verbandsbildung und Beitrittsmotiven liegen nur vereinzelt vor (zusammenfassend: Traxler 1999). Sie sind zudem implizit oder explizit an

25 Die Wirtschaftsverbände nehmen neben ihren Außen- auch Binnenaufgaben wahr (Rampelt 1979: 24-32). Diese Funktionen werden im ÖD weitgehend intern durchgeführt.

26 Der Organisationsgrad kann innerhalb der einzelnen Länder unterschiedlich sein. Die größeren Kommunen (mit über 20.000 Einwohnern) sind alle organisiert, ebenso ein beachtlicher Teil derjenigen mit 10.000-20.000 Einwohnern, so dass der Organisationsgrad gemessen an der Anzahl der von den Verbandsmitgliedern beschäftigten Arbeitnehmer höher liegt, als wenn man ihn an der reinen Zahl der Arbeitgeber messen würde. 
den Rahmenbedingungen der Privatwirtschaft orientiert und daher kaum auf den ÖD anzuwenden. Am ehesten eignet sich die Theorie des kollektiven Handelns von Olson (1968), vor allem wenn man sie auf die kommunale Ebene bezieht.

Olson betont wesentliche Unterschiede zwischen kleinen und großen Gruppen. Er zeigt, dass sich Individuen in großen bzw. latenten Gruppen keinesfalls spontan zusammenschließen, da es sich bei den zu erwartenden Vorteilen um öffentliche Güter handelt, zu deren Erstellung kaum freiwillige Beiträge geleistet werden. Es bedarf vielmehr besonderer Bedingungen vor allem in Form von Zwang oder selektiven Anreizen, damit rationale und eigeninteressiert handelnde Individuen die Erreichung gemeinsamer Ziele tatsächlich durch eigene Beiträge fördern.

Große Gruppen sind im Gegensatz zu kleinen dadurch charakterisiert, dass keine spürbaren Interdependenzen zwischen den Handlungen der Individuen festzustellen sind. Wegen der fehlenden Wahrnehmbarkeit bzw. wechselseitigen Abhängigkeit besteht für Individuen in großen Gruppen kein Anlass, zur Erstellung von Kollektivgütern beizutragen:

- die individuellen Beiträge bleiben unmerklich,

- bei zunehmender Gruppengröße wird es immer kostspieliger und damit praktisch unmöglich, Nichtmitglieder vom Konsum auszuschließen,

- daher gibt es keine Anreize für wechselseitige Verhaltenskontrollen.

Aus diesem Zusammenhang von Gruppengröße und individuellem Handeln resultiert das für Organisationen typische Free-Rider-Problem (,Trittbrett- oder Schwarzfahrerproblem"): Individuen beteiligen sich nicht an den Kosten der Erstellung des Kollektivgutes, ohne von dessen Nutzung ausgeschlossen werden zu können.

Die eine Hauptaufgabe der Verbände, der Abschluss von Tarifverträgen, stellt ein Kollektivgut dar, von dessen Erstellung auch Nichtmitglieder profitieren können. Bei großen Gruppen wie den KAV muss deshalb ein Verband, der auf freiwilliger Mitgliedschaft beruht und keinen Zwang im Sinne von Olson ausüben kann, selektive Anreize zum Beitritt zur Verfügung haben. Aus der umfangreichen Forschung vor allem über Gewerkschaften (Crouch 1982: $51 \mathrm{ff}$.), aber auch über Arbeitgeberverbände der Privatwirtschaft (Rampelt 1979: 34 ff., Gladstone 1984: $29 \mathrm{ff}$.) wissen wir, dass diese Verbände eine breite Palette von privaten Gütern anbieten, „zu denen u. a. die Beratung in arbeits- und sozialrechtlichen Angelegenheiten sowie diverse Informationsdienste (z. B. zur wirtschaftspolitischen Entwicklung) zählen (...)“(Traxler 1985: 56).

Ähnlich ist der Sachverhalt bei den KAV: Ihre Hauptaktivitäten sind die Mitwirkung bei der Gestaltung der Tarifpolitik, also bei der Erstellung kollektiver Güter, sowie die Beratung und Information der Mitglieder, also die Produktion privater Güter. Da die zuletzt genannten Aufgaben im Rahmen der allgemein feststellbaren Veränderungen von der Einfluss- zur Mitgliedslogik zugenommen haben, nehmen KAV den Charakter von Dienstleistungsunternehmen an:

- Die Mitglieder haben satzungsgemäß Anrecht auf schriftliche und/oder mündliche Beratung durch Referenten bzw. Sachbearbeiter des Verbandes bei allen tarif- und arbeitsrechtlichen Problemen; sie machen von dieser Möglichkeit regen Gebrauch, was zu einer hohen zeitlichen Inanspruchnahme des Verbandsapparats führt. 
- Mit diesem satzungsrechtlich garantierten Anspruch auf Beratung verbunden ist die Hilfestellung des Verbandes bei Rechtsstreitigkeiten. Die Prozessvertretung ${ }^{27}$ vor Arbeitsgerichten (einschließlich Landes- und Bundesarbeitsgericht), Sozialgerichten (einschließlich Landes- und Bundessozialgericht), Verwaltungs- sowie dem Oberverwaltungsgericht erstreckt sich auf das gesamte Gebiet des Arbeits- und Tarifrechts, wobei Kündigungsschutz- und Eingruppierungsprobleme dominieren. Ähnlich wie bei Arbeitgeberverbänden der Privatwirtschaft ist diese Prozessvertretung vor allem für kleinere Mitglieder wichtig, die im Gegensatz zu großen über keine eigene Rechtsabteilung verfügen.

- Weiterhin informiert die Geschäftsstelle des Verbandes die Mitglieder durch Rundschreiben über relevante Entwicklungen vor allem des Tarif- und Arbeitsrechts. Dabei sind vom Adressatenkreis her zu unterscheiden: allgemeine Rundschreiben, die an alle Mitglieder gehen, und Sonderrundschreiben, die sich an einzelne Verbandsgruppen richten. Die allgemeinen Informationen, die eine Größenordnung von ca. 100 pro Jahr und Verband erreichen können, sind häufiger als die speziellen. Die Differenzierung soll eine Überfrachtung der Mitglieder mit Informationen verhindern.

Im Sinne der Analyse von Olson ist einer der ältesten, in der Literatur wiederholt analysierten selektiven Anreize zum Verbandsbeitritt die Unterstützung bei Arbeitskämpfen. Dieses private Gut stellen in der Privatwirtschaft vor allem Gewerkschaften, aber auch Arbeitgeberverbände für ihre Mitglieder bereit. Bei den Arbeitgeberverbänden des ÖD gibt es keine derartigen Einrichtungen in Form sogenannter Gefahrengemeinschaften der Tarifvertragsverbände, so dass einer der wichtigeren Anreize nicht zur Verfügung steht. Allerdings sind Streiks im ÖD sehr selten und Aussperrungen kommen nicht vor, so dass dieser Unterschied faktisch nicht bedeutend ist.

Neben den Beziehungen zwischen KAV und Einzelmitgliedern ist das Verhältnis zwischen Spitzenorganisation und Mitgliedsverband zu analysieren. Die Mitglieder haben gegenüber der VKA u. a. Rechte auf

- Beratung in allen Fragen, die den Aufgabenbereich der VKA tangieren,

- Unterrichtung über Tätigkeiten der VKA und wichtige Ereignisse, insbesondere über gewerkschaftliche Forderungen und Kündigungen von Tarifverträgen,

- Teilhabe an Dienstleistungen und Einrichtungen der VKA (VKA 2003: § 5). Die VKA verfasst Stellungnahmen zu arbeitsrechtlichen Problemen und unterhält einen eigenen Urteilsdienst zur aktuellen Rechtsprechung für die Mitglieder; Statistiken werden nur über Personalkosten erstellt.

Zwischen den Mitgliedsverbänden und Spitzenorganisationen bestehen permanente schriftliche und telefonische Kontakte. Für die innerverbandliche Verhaltenskoordination sorgt vor allem die Geschäftsführerkonferenz, der die hauptamtlichen Geschäftsführer der Mitgliedsverbände sowie der Hauptgeschäftsführer der VKA angehören. Diese personelle Zusammensetzung garantiert, dass alle Interessen im Abstimmungsprozess Berücksichtigung finden. Die Geschäftsführerkonferenz behandelt Probleme von herausragender Bedeu-

27 Die Prozessvertretung der Mitglieder erfolgt entweder ab der zweiten Instanz, bei Fragen von grundsätzlicher Bedeutung auch von der ersten Instanz an oder prinzipiell von der ersten Instanz an. Im Übrigen führen nur die Mitgliedsverbände, nicht dagegen die Spitzenorganisation Rechtsstreitigkeiten. 
tung bzw. allgemeinem Interesse, u. a. tarifpolitische Fragen; sie hat „den Informations- und Erfahrungstausch zu fördern und zur Auslegung von Gesetzen und Tarifverträgen sowie von Richtlinien und Beschlüssen der Organe der VKA Stellung zu nehmen, um die einheitliche Anwendung zu gewährleisten“ (VKA 2003: § 17).

Anders als bei den KAV und der VKA liegt das Kollektivgutproblem bei der TdL, die im Gegensatz zu Arbeitgeberverbänden kaum Serviceleistungen für ihre Mitglieder anbietet - und daher nur über wenige Mitarbeiter verfügt. Ähnlich liegt der Sachverhalt bei den zuständigen Abteilungen der Ministerien der Bundesländer. Rechtsberatung spielt keine Rolle, allenfalls werden bestimmte Informationsleistungen erbracht wie die Entwicklung der Personalkosten oder des Sozialprodukts. Im Übrigen ist auch im ÖD häufig juristischer Sachverstand gefragt; das Personal ist fachlich hoch qualifiziert (hoher Akademikeranteil). In der Terminologie von Olson (1968) handelt es sich bei der TdL um eine kleine Gruppe, die kaum Probleme bei der Bereitstellung von Kollektivgütern haben sollte. Insofern ist die Konzentration auf die Führung von Tarifverhandlungen innerverbandlich unproblematisch.

\section{Aktuelle Entwicklungen und Perspektiven}

\subsection{Strukturen der Kollektivverhandlungen}

Das Tarifverhandlungssystem des ÖD ist im Gegensatz etwa zu denen in angelsächsischen Ländern, aber ähnlich wie u. a. in den skandinavischen Ländern durch weitgehende Zentralisierung und Integration gekennzeichnet (international vergleichend: Bach et al. 1999; Dell'Aringa et al. 2001). Dabei werden Unterscheidungen weder nach den drei Ebenen (Bund, Länder, Gemeinden) noch nach Statusverhältnisssen (Arbeiter und Angestellte) vorgenommen (,multi-employer bargaining"). Eine von den korporativen Akteuren durchaus beabsichtigte Folge ist eine hochgradige Standardisierung der Entgelte und übrigen Arbeitsbedingungen, was in Anbetracht der föderalistischen Verfassung der Bundesrepublik nicht selbstverständlich ist. Diese weitgehende Vereinheitlichung liegt auch im Interesse der Gewerkschaften, die dadurch Legitimationsprobleme gegenüber ihren Mitgliedern eher lösen können (zusammenfassend: Keller 1999). Ein weiteres Resultat sind sehr hohe Deckungsraten, d.h. ein hoher Anteil der Arbeitnehmer wird von Kollektivverträgen erfasst, sowohl in Bezug auf die Arbeitgeber als auch auf die bei ihnen Beschäftigten. Sogenannte stille Tarifflucht, d.h. ein faktisches Unterbieten vereinbarter tariflicher Standards trotz Verbandsmitgliedschaft, kommt im Gegensatz zur Privatwirtschaft nicht vor; insofern ist die Verpflichtungsfähigkeit der Verbände nach wie vor gegeben.

Bei traditionell engen und kooperativen Beziehungen zwischen den Arbeitgeberverbänden gelingt normalerweise die Ex-ante-Koordination der Interessen in prozedural schwierigen Willensbildungs- und Abstimmungsprozessen, so dass gemeinsames und solidarisches Handeln möglich wird. ${ }^{28}$

„Diese allerdings nicht institutionalisierte Zusammenarbeit hat sich nach übereinstimmender Ansicht für den Bund, die TdL und die VKA gut bewährt; denn die drei ,öffentlichen Arbeitge-

28 Falls Bewertungsunterschiede auftreten, verfahren die Beteiligten häufig nach dem Prinzip der relativen Betroffenheit: Sie akzeptieren die Position des am stärksten betroffenen Bereichs als Mehrheitsmeinung. 
ber` haben dadurch, insbesondere den Gewerkschaften gegenüber, zwangsläufig ein größeres Gewicht. Gegenseitige Präjudizierungen werden vermieden“(Berger 1980: 221).

Alle Arbeitgeber haben nach übereinstimmender Beurteilung den Wert einheitlicher Tarifverhandlungen erkannt und wollen diese unter keinen Umständen aufgeben, da in getrennten Verhandlungen ihre Position durch tarifpolitische Zersplitterung geschwächt würde. Der informelle Charakter der Abstimmungsprozesse erhöht die Flexibilität der Verhandlungsführung. ${ }^{29}$ Die Tatsache, dass ein der BDA entsprechender Dachverband zur tarif- und sozialpolitischen Interessenwahrnehmung formal für den ÖD nicht besteht, stellt in Anbetracht der faktisch engen Kooperation keinen gravierenden Nachteil dar.

Die partiell unterschiedlichen Interessen der Vertreter der drei Arbeitgeber ${ }^{30}$ führen zu unterschiedlichen Verhaltensformen bei der internen Willensbildung vor und während der Tarifverhandlungen. Charakteristisch für diese Prozesse ist ihre Zweistufigkeit, da sie sich sowohl innerhalb als auch zwischen Gruppen vollziehen. Willensbildungsprozesse zwischen Gruppen beeinflussen das Verhandlungsverhalten und -ergebnis stärker als die innerhalb einer Gruppe. Unterschiedliche Bewertungen, auf die Rücksicht genommen werden muss, ergeben sich nicht so sehr zwischen den Vertretern des Bundes und der Länder als vielmehr im Verhältnis zu den Vertretern der Kommunen. ${ }^{31}$ Die Bestimmungsgründe für diese Differenzen liegen in der schlechten finanziellen Situation sowie der besonderen Streikanfälligkeit der Kommunen. Echte Interessenkonflikte allgemeiner Art (niedriger Abschluss versus politisches Überleben) sowie solche zwischen den Vertretern der Gemeinden, der Länder und des Bundes sind selten (zu den Ausnahmen: Keller 1983).

Insofern liegt eine andere Situation vor als bei Arbeitgeberverbänden der Privatwirtschaft. Der Unterschied besteht darin, dass die Arbeitgeber des ÖD sowohl auf den Arbeitsals auch auf den Produktmärkten nur in begrenztem Maße in einem Konkurrenzverhältnis zueinander stehen; dadurch treten Probleme der Herstellung innerverbandlicher Solidarität weniger stark auf als bei den Arbeitgeberverbänden in der Privatwirtschaft.

Das Dilemma „zwischen Kompromisserreichung und Verpflichtung der eigenen Organisation" (Weitbrecht 1973: 22) wird ähnlich wie in der Privatwirtschaft gelöst: Die Beschlussorgane, die den Großen Tarifkommissionen der Gewerkschaften entsprechen, sind auf Seiten der Arbeitgeber die Mitgliederversammlungen von VKA und TdL sowie die Bundesregierung. Diese Gremien werden während der Verhandlungen wiederholt von ihren Repräsentanten informell konsultiert und über den aktuellen Verlauf sowie über Modifikationen der unterschiedlichen Positionen und alternative Möglichkeiten eines Abschlusses informiert. Sie müssen später dem ausgehandelten Kompromiss formal und mit qualifizierten Mehrheiten zustimmen, wodurch die Folgebereitschaft der Mitglieder gesichert wird. Während die Mitgliederversammlung der VKA in den wichtigen Phasen in ihren Entscheidungen autonom ist, hat bei der TdL die politische Ebene wesentlichen Einfluss auf den Prozess der Willensbildung.

29 Im Prozess der Entscheidungsfindung wird die Kommission immer weiter verkleinert, was die Kompromissfindung erleichtert: An der ersten Verhandlungsrunde, bei der die sogenannten „Fensterreden“ gehalten werden, sind mehr als 200 Personen beteiligt. Später tagt dann die sogenannte „48er Kommission“. In der entscheidenden Phase erarbeitet der sogenannte „,kleine Kreis“ („,6er Kreis“) einen einigungsfähigen Kompromiss.

30 Divergierende Interessen können z.B. dadurch auftreten, dass die Gewerkschaftsforderungen wie in den späten 1960er und 1970er Jahren sogenannte soziale Komponenten enthalten, von denen die Arbeitgeber wegen ihrer differierenden Personalstrukturen in unterschiedlichem Ausmaß betroffen sind (Keller 1983).

31 Das innerorganisatorische Bargaining (Walton/McKersie 1991) ist auch auf Arbeitgeberseite recht komplex. 


\subsection{Aktuelle Entwicklungen}

Aus den Verbandssatzungen, die Integrations- und Legitimationsfunktionen haben, ergeben sich Pflichten der Mitglieder wie die Durchführung abgeschlossener Tarifverträge und sonstiger Vereinbarungen, ein weitgehender Verzicht auf den selbständigen Abschluss von Tarifverträgen sowie ein Verbot der Unterbietung oder Überschreitung von Tarifvertragsbedingungen. Die Ahndung von Verstößen der Mitglieder bzw. bei Spitzenorganisationen der Mitgliedsverbände gegen diese Pflichten kann durch Verbandsstrafen in Form von Geldbußen oder in gravierenden Fällen durch Ausschluss erfolgen (Bunn 1984: $189 \mathrm{ff}$. . $^{32}$ Allerdings ist diese verbandsautonome Sanktionierung eine selten praktizierte, allenfalls ergänzende Kontrollleistung, wobei ein Ausschluss noch seltener als eine Geldbuße verhängt wird. Insofern besteht kein wesentlicher Unterschied zu Arbeitgeberverbänden der Privatwirtschaft (Rampelt 1979: 59; Schmitter/Streeck 1981: 233 ff.).

Solidarität im Binnenverhältnis der Mitglieder, vor allem die Befolgung der Tarifgebundenheit, ist eine notwendige Voraussetzung für Handlungs- und Entscheidungsfähigkeit im Außenverhältnis, d.h. gegenüber dem Tarifpartner. Solidarisches Handeln muss bei einer Mitgliedschaft, die wegen der grundgesetzlich garantierten Vereinigungsfreiheit freiwillig ist, durch andere Mechanismen garantiert werden als durch den Einsatz der dem Verband autonom zur Verfügung stehenden Zwangsmittel, vor allem durch freiwillige Übereinkünfte aller Beteiligten. Im Übrigen besteht im ÖD für den Fall einer Realisierung der ExitOption (Hirschman 1974) keine Alternative, d.h. kein konkurrierender Verband; ein Austritt ist gleichbedeutend damit, nicht organisiert zu sein.

In dieser Hinsicht existieren zwei Besonderheiten: Die TdL schloss 1994 das Land Berlin aus, weil es gegen Grundsätze der TdL verstieß, indem es im Ostteil Entgeltregelungen für das Tarifgebiet West anwandte. Berlin schloss im Frühjahr 2003 eigenständige Tarifverträge mit den zuständigen Gewerkschaften. Außerdem trat das Land Hessen im März 2004 aus der TdL aus (http://www.tdl.bayern.de). Diese „Organisationskrise“ der TdL ist prima facie überraschend, weil - zumindest in der Olson'schen Rational-ChoicePerspektive - Konflikte aufgrund der großen Mitgliederzahl am ehesten bei den Verbänden der kommunalen Ebene zu erwarten wären. Eine Erklärung gelingt am ehesten, wenn man die Dominanz der finanziellen Rahmenbedingungen in den Mittelpunkt rückt und nicht andere, etwa parteipolitische Kalküle. Bei den Ländern ist aufgrund der grundgesetzlich vorgegebenen Aufgabenverteilung - und nicht wegen ihrer spezifischen Haushaltsund oder Personalpolitiken - der Anteil der Personal- an den Gesamtausgaben traditionell wesentlich höher als bei den Kommunen (Keller 2006). Erstaunlich ist insofern nicht die Tatsache des Austritts der Länder aus der Verhandlungsgemeinschaft, sondern der späte Zeitpunkt.

Die etablierten, hochgradig zentralisierten Strukturen der Kollektivverhandlungen ${ }^{33}$ zerfielen nach der Tarifrunde des Jahres 2003 (Behrens 2003), als einige unionsregierte Bundesländer (u. a. Baden-Württemberg, Hessen, Niedersachsen) nach einem als zu hoch empfundenen Abschluss mit Austritt aus der TdL drohten, um eigenständige Regelungen in

32 „Der Ausschluss selbst stellt für den Verband eine nur wenig attraktive Option dar, da er Integration nur durch erzwungene Desintegration aufrechterhält. Infolge der Abhängigkeit ihrer Wirksamkeit von jener normativer und/oder remunerativer Integrationsmodi können die den Verbänden autonom zur Verfügung stehenden Zwangsmittel nur ergänzende Kontrollleistungen sein.“( Traxler 1980: 13).

33 Erste Erosionstendenzen zeigten sich vorher bereits im Abschluss einiger sogenannter Spartentarifverträge (u. a. im Personennahverkehr); Privatisierungsmaßnahmen wirken in dieselbe Richtung. 
einigen Bereichen (wie Sonderzahlungen) einführen zu können. Daraufhin beschloss die TdL nach internen Auseinandersetzungen, die Mitgliedschaft in der Verhandlungsgemeinschaft der Arbeitgeberseite aufzukündigen und derartige Koalitionen in Zukunft nur noch auf Ad-hoc-Basis als „situative Zweckbündnisse“ einzugehen (Dribbusch 2003).

Anfang 2005 fanden die ersten getrennten Tarifverhandlungen zwischen Gewerkschaften (Vereinte Dienstleistungsgewerkschaft - ver.di ${ }^{34}$ und Tarifunion des DBB) und den Arbeitgebern von Bund und Kommunen statt. Nachdem die Bundesländer die Tarifverträge über Arbeitszeiten gekündigt hatten, brach ver.di wegen dieses Schrittes die Gespräche über die Ausgestaltung eines neuen Tarifrechts ab und erklärte deren Scheitern; die Bundesländer nahmen an den folgenden Verhandlungen nicht teil. Nach Abschluss der Verhandlungsrunde verweigerten sich die Länder aus Gründen finanzieller Mehrbelastungen kategorisch den wiederholten Forderungen der Gewerkschaft, den Abschluss „eins zu eins“ zu übernehmen, bestanden auf unabhängigen Verhandlungen bzw. „strikter Kostenneutralität“ und forderten die Vereinbarung von Öffnungsklauseln für Regelungen zu Arbeitszeiten und Sonderzahlungen (Weihnachts- und Urlaubsgeld). Streikdrohungen bzw. einzelne Streiks waren nicht in der Lage, die Einheitlichkeit der Arbeitsbedingungen wiederherzustellen.

Nach dieser als weitreichend zu bezeichnenden Zäsur sind zwei Szenarien im Rahmen eines ,institution building“ möglich: Entweder verhandeln die Bundesländer zukünftig gemeinsam, aber getrennt von Bund und Kommunen oder die Länder verhandeln nicht nur unabhängig von Bund und Kommunen, sondern auch getrennt voneinander (etwa nach Ost und West bzw. finanzschwachen und -starken Ländern). Die zuerst genannte Option einer ausschließlich vertikalen Differenzierung nach Ebenen, wäre gleichbedeutend mit dem Fortbestand der TdL, die ein Eigeninteresse an dieser Alternative haben muss, sowie mit einer moderaten, kontrollierten Dezentralisierung. Die zuletzt genannte Option hingegen würde eine zusätzliche horizontale Differenzierung mit einer faktischen Auflösung bzw. Bedeutungslosigkeit der TdL und eine deutlich weiter gehende Dezentralisierung (im Sinne einer gewissen Regionalisierung) bedingen. Fraglich ist nicht, ob die Dezentralisierung des zentralisierten Bargaining-Systems fortgesetzt wird, sondern welches Ausmaß sie annehmen wird. Selbst im weiter gehenden Fall wäre sie noch weit von einer sogenannten Verbetrieblichung entfernt.

Die mittel- und langfristigen Konsequenzen ihres Austritts aus der Verhandlungsgemeinschaft sind für die Bundesländer keinesfalls eindeutig: Ob sie in getrennten Verhandlungen tatsächlich günstigere Abschlüsse als in gemeinsamen Verhandlungen mit Bund und Kommunen durchsetzen können, hängt wesentlich von der Verhandlungsmacht sowie den Ressourcen der Gewerkschaft ab. ${ }^{35}$ Die anderen korporativen Akteure sind jedenfalls nicht sonderlich an Regelungen interessiert, die nach den drei Ebenen unterschiedlich ausfallen. Sie würden dem bisher weitgehend akzeptierten Prinzip der Einheitlichkeit von Arbeitsund damit Lebensbedingungen im ÖD widersprechen. Eine weiter gehende. materielle Kon-

34 Bis zum Zusammenschluss von fünf Einzelgewerkschaften zu ver.di im Frühjahr 2001 war die Gewerkschaft Öffentliche Dienste, Transport und Verkehr (ÖTV) Verhandlungsführerin; zu den kleineren DGBMitgliedsgewerkschaften, die ebenfalls im ÖD organisierten (GEW, GdP, DPG und GdED), bestanden traditionell enge informelle Kontakte (Keller 1993).

35 Eine aufgrund von Forderungen der Arbeitgeber in den TVöD integrierte, in der Öffentlichkeit weitgehend unbeachtete sogenannte Meistbegünstigungsklausel besagt: Sollten die Länder einen für sie vorteilhafteren Abschluss (etwa längere Arbeitszeiten) erzielen, gilt dieser ,als unwiderrufliches Angebot an den Bund und die Kommunen, diese Regelungen zu übernehmen“. Die Vereinbarung dieser Klausel erhöht auf Gewerkschaftsseite den Widerstand gegen abweichende Abschlüsse mit den Ländern (Keller 2006). 
sequenz wäre eine Verschlechterung der Arbeitsbedingungen (Entgelte, Arbeitszeiten) der im ÖD Beschäftigten.

\section{Literatur}

Bach, Stephen/Bordogna, Lorenzo/Della Rocca, Guiseppe/Winchester, David (Hrsg.) (1999): Public Service Employment Relations in Europe. Transformation, Modernization or Inertia. London: Routledge.

Behrens, Martin (2003): New Agreement Signed in Public Sector. In: EIROnline. European industrial relations observatory on-line, 01. URL: http://www.eurofound.europa.eu/eiro/2003/01/feature/ de0301204f.htm (zuletzt besucht am 7.12.2009).

Behrens, Martin/Traxler, Franz (2004): Employers' Organisations in Europe. In: Eiro-observer, 3, S. i-viii.

Berger, Jakob (1980): Die Kommunen als Arbeitgeber. In: Deutscher Städtetag (Hrsg.): Im Dienst deutscher Städte 1905-1980. Ein kommunales Sachbuch zum 75jährigen Jubiläum. Stuttgart: Kohlhammer, S. 217-222.

Bunn, Ronald F. (1984): Employers Associations in the Federal Republic of Germany. In: Windmuller, John P./Gladstone, Alan (Hrsg.): Employers Associations and Industrial Relations. Oxford: Clarendon Press, S. 169-201.

Crouch, Colin (1982): Trade Unions. The Logic of Collective Action. Glasgow: Fontana.

Dell'Aringa, Carlo/Della Rocca, Guiseppe/Keller, Berndt (Hrsg.) (2001): Strategic Choices in Reforming Public Service Employment. An International Handbook. Basingstoke: Palgrave.

Dribbusch, Heiner (2003): Public Sector Employers' Bargaining Association Collapses. In: EIROnline. European industrial relations observatory on-line, 06. URL: http://www.eurofound.europa.eu/ eiro/2003/06/inbrief/de0306202n.htm (zuletzt besucht am 7.12.2009).

Gladstone, Alan (1984): Employers Associations in Comparative Perspective: Functions and Activities. In: Windmuller, John P./Gladstone, Alan (Hrsg.): Employers Associations and Industrial Relations. Oxford: Clarendon Press, S. 24-43.

Henneberger, Fred (1997): Arbeitsmärkte und Beschäftigung im öffentlichen Dienst. Eine theoretische und empirische Analyse für die Bundesrepublik Deutschland. Bern/Stuttgart: Haupt.

Henneberger, Fred (2004): Studien zum Arbeitsmarkt. Bern/Stuttgart: Haupt.

Hirschman, Albert O. (1974): Abwanderung und Widerspruch. Reaktionen auf Leistungsabfall bei Unternehmungen, Organisationen und Staaten. Tübingen: Mohr.

Keller, Berndt (1983): Arbeitsbeziehungen im öffentlichen Dienst. Tarifpolitik der Gewerkschaften und Interessenpolitik der Beamtenverbände. Frankfurt a. M./New York: Campus.

Keller, Berndt (1987a): Kommunale Arbeitgeber und ihre Verbände. Zur sozialwissenschaftlichen Analyse einer „Forschungslücke“. In: Zeitschrift für öffentliche und gemeinwirtschaftliche Unternehmen, 10, S. 262-287.

Keller, Berndt (1987b): Interessenaggregation und -transformation in Verbänden öffentlicher Arbeitgeber. In: Windhoff-Héritier, Adrienne (Hrsg.): Verwaltung und ihre Umwelt. Festschrift für Thomas Ellwein. Opladen: Westdeutscher Verlag, S. 258-276.

Keller, Berndt (1993): Arbeitspolitik des öffentlichen Sektors. Baden-Baden: Nomos.

Keller, Berndt (1999): Germany. Negotiated Change, Modernization and the Challenge of Unification. In: Bach, Stephen/Bordogna, Lorenzo/Della Rocca, Guiseppe/Winchester, David (Hrsg.): Public Service Employment Relations in Europe. Transformation, Modernization or Inertia. London: Routledge, S. 56-93.

Keller, Berndt (2006): Aktuelle Entwicklungen der Beschäftigungsbeziehungen im öffentlichen Dienst. In: Die Verwaltung, 39, S. 79-99.

Keller, Berndt/Henneberger, Fred (1999): Privatwirtschaft und öffentlicher Dienst: Parallelen und Differenzen in den Arbeitspolitiken. In: Müller-Jentsch, Walther (Hrsg.): Konfliktpartnerschaft. 
Akteure und Institutionen der industriellen Beziehungen. München/Mering: Hampp (3., überarb. u. erw. Aufl.), S. 233-256.

Keller, Berndt/Schnell, Rainer (2003): Zur empirischen Analyse von Personalräten - Strukturdaten und Probleme der Interessenvertretung. In: WSI-Mitteilungen, 56, S. 185-193.

Keller, Berndt/Schnell, Rainer (2005): Sozialstruktur und Problemfelder der Interessenvertretung im öffentlichen Dienst. Eine empirische Untersuchung von Personalräten in West- und Ostdeutschland. In: Berliner Journal für Soziologie, 15, S. 87-102.

McPherson, William (1971): Public Employee Relations in West Germany. Ann Arbor: Institute of Labor and Industrial Relations. The University of Michigan - Wayne State University.

Naschold, Frieder/Bogumil, Jörg (2000): Modernisierung des Staates: New Public Management in deutscher und internationaler Perspektive. Opladen: Leske + Budrich (2., vollst. aktual. u. erw. Aufl.).

Olson, Mancur (1968): Die Logik des kollektiven Handelns. Tübingen: Mohr Siebeck.

Olson, Mancur (1982): The Rise and Decline of Nations. Economic Growth, Stagflation, and Social Rigidities. New Haven: Yale University Press.

Rampelt, Jörg (1979): Zur Organisations- und Entscheidungsstruktur in westdeutschen Unternehmerverbänden. Ein Literaturbericht. WZB Discussion Paper IIM DP 79-111. Berlin: Wissenschaftszentrum Berlin für Sozialforschung (WZB).

Rosdücher, Jörg (1994): Kommunale Arbeitgeberverbände in den neuen Bundesländern. In: Zeitschrift für öffentliche und gemeinwirtschaftliche Unternehmen, 17, S. 414-429.

Schmitter, Phillippe C./Streeck, Wolfgang (1981): The Organization of Business Interests. A Research Design to Study the Associative Action of Business in the Advanced Industrial Societies of Western Europe. Revised and extended version, August 1981. WZB Discussion Paper IIM LMP 81-13. Berlin: Wissenschaftszentrum Berlin für Sozialforschung (WZB).

TdL (2005): Satzung der Tarifgemeinschaft deutscher Länder (Stand: 21. Februar 2005). Bonn: TdL.

Traxler, Franz (1980): Unternehmerverbände und Gewerkschaften: Bedingungen kollektiver Handlungsfähigkeit. In: Journal für Sozialforschung, 20, S. 7-25.

Traxler, Franz (1985): Arbeitgeberverbände. In: Endruweit, Günter/Gaugler, Eduard/Staehle, Wolfgang H./Wilpert, Bernhard (Hrsg.): Handbuch der Arbeitsbeziehungen. Deutschland, Österreich, Schweiz. Berlin/New York: de Gruyter, S. 51-64.

Traxler, Franz (1999): Gewerkschaften und Arbeitgeberverbände: Probleme der Verbandsbildung und Interessenvereinheitlichung. In: Müller-Jentsch, Walther (Hrsg.): Konfliktpartnerschaft. Akteure und Institutionen der industriellen Beziehungen. München/Mering: Hampp (3., überarb. und erw. Aufl.), S. 57-77.

VKA (2003): Satzung der Vereinigung der kommunalen Arbeitgeberverbände (VKA) (Stand: 1. Januar 2003). Köln: VKA.

Walton, Richard E./McKersie, Robert B. (1991): A Behavioral Theory of Labor Negotiations. An Analysis of a Social Interaction System. New York: McGraw-Hill (2. Aufl.).

Weitbrecht, Hansjörg (1969): Effektivität und Legitimität der Tarifautonomie. Berlin: Duncker \& Humblot.

Weitbrecht, Hansjörg (1973): Wirkung und Verfahren der Tarifautonomie. Ein soziologischer Vergleich zum Konflikt der Tarifpartner in Wirtschaft und öffentlichem Dienst. Baden-Baden: Nomos Verlagsgesellschaft.

Windmuller, John P. (1984): Employers Associations in Comparative Perspective: Organization, Structure, Administration. In: Windmuller, John P./Gladstone, Alan (Hrsg.): Employers Associations and Industrial Relations. Oxford: Clarendon Press, S. 1-23.

Windmuller, John P. (1987): Comparative Study of Methods and Practices. In: ILO (Hrsg.): Collective Bargaining in Industrialised Market Economies. A Reappraisal. Geneva: ILO, S. 3-160. 